Article

\title{
Potential Synergy between Spores of Metarhizium anisopliae and Plant Secondary Metabolite, 1-Chlorooctadecane for Effective Natural Acaricide Development
}

\author{
Abid Hussain 1,2,3 (D) and Ahmed Mohammed AlJabr 1,* \\ 1 Laboratory of Bio-Control and Molecular Biology, Department of Arid Land Agriculture, College of \\ Agricultural and Food Sciences, King Faisal University, Hofuf 31982, Al-Ahsa, Saudi Arabia; \\ solvia_aah@yahoo.com \\ 2 Research and Consulting Institute, King Faisal University, Hofuf 31982, Al-Ahsa, Saudi Arabia \\ 3 Ministry of Environment, Water and Agriculture, Riyadh 11442, Saudi Arabia \\ * Correspondence: aljabr@kfu.edu.sa; Tel.: +9-6650-691-4442
}

Academic Editor: Giovanni Benelli

Received: 4 April 2020; Accepted: 18 April 2020; Published: 20 April 2020

\begin{abstract}
Date palm dust mites are important pests severely infesting valuable nutritious fruits (dates) of date palm. In search of an alternative to acaricides, joint action of Metarhizium anisopliae EBCL 02049 spores and 1-Chlorooctadecane was evaluated as a potential candidate for the management of Oligonychus afrasiaticus through natural products. In this regard, in vitro tests were performed to evaluate the interaction of M. anisopliae spores with multiple doses of 1-Chlorooctadecane ( 0.8 , $1.6,2.4,3.2$, and $4.0 \mathrm{mg} / \mathrm{mL}$ ). Compatibility bioassay results evidenced from vegetative growth $(77.7-84.40 \mathrm{~mm})$, sporulation $\left(5.50-7.30 \times 10^{6}\right.$ spores $\left./ \mathrm{mL}\right)$, and germination $(96.70-98.20 \%)$, revealed that all the tested doses are compatible (biological index > 82) with the spores of M. anisopliae. The impact of combined treatment of spores with 1-Chlorooctadecane in different proportions (Scheme I, II, III, and IV) compared to their sole application against O. afrasiaticus was evaluated by concentration-mortality response bioassays. Results showed that all the combined treatments revealed high mortality compared to the sole application, which showed relatively slow mortality response over time. Toxicity recorded from Scheme IV combinations (80\% 1-Chlorooctadecane: $20 \%$ Spores), exhibited strong synergistic interaction (joint toxicity $=713$ ). Furthermore, potent interactions have overcome the host antioxidant defense at the final stage of infection by tremendously reducing catalase, and superoxide dismutase activities. These experiments demonstrated fungal-toxin joint synergistic interaction as a promising date palm dust mite management option.
\end{abstract}

Keywords: antioxidants; compatibility; date palm dust mites; host defense; Oligonychus afrasiaticus; Metarhizium anisopliae; natural acaricide; synergism; 1-Chlorooctadecane

\section{Introduction}

Date palm (Phoenix dactylifera $\mathrm{L}$.) is one of the oldest domesticated fruit trees with unique nutritional characteristics and great socioeconomic importance for the Middle East and North African countries. This cash crop, in addition to commercial and nutritional values, can successfully be grown in the deserts because of minimum water requirement and highly tolerance to salinity and harsh weather conditions [1]. In the Kingdom of Saudi Arabia, date palms are grown on vast areas of land, accounting for about $25 \%$ of the World's date palms ( 30 million). According to an estimate, approximately 450 different cultivars out of 2000 are grown here in Saudi Arabia [2]. 
The economic importance of date palms is mainly because of valuable nutritious fruit (dates), which contains carbohydrates, dietary fibers, fat, lipids, minerals and protein [3]. Saudi Arabia stands second (1,302,859 tons), after the major producer Egypt (1,562,171 tons), in the world in the production of dates [4]. However, the annual yield of dates in Saudi Arabia compared to the other countries is still low might because of pest infestations. Date palm dust mites, O. afrasiaticus (Acari: Tetranychidae) is considered a major pest of date palms in Saudi Arabia. The weather conditions prevailing in the Kingdom and neighboring countries including Qatar, Sultanate of Oman, United Arab Emirates, Yemen, Libya, Iraq, and Egypt greatly favor O. afrasiaticus growth and development.

The infestation of $O$. afrasiaticus mainly depends on the moisture contents of the dates. The attack of mites starts at the Kimri stage (greenish), during this stage these greenish dates tremendously increase in sugar contents and moisture level that ultimately led to an increase in size and weight. At this stage, mites start spinning the web around the bunches of dates. Dust particles and exuviae from different stages are trapped into these webs resulting in the dusty appearance of bunches. Under these conditions, mites grow rapidly and multiply logarithmically [5].

Currently, management of date palm dust mites heavily relied on the use of acaricides. The use of synthetic pesticides to manage mites on dates may cause serious health hazards because the fruit contains pesticide residues. Furthermore, the use of pesticides is discouraged because of (1) environmental pollution, (2) applicator safety issues, (3) detrimental effects on non-target animals and (4) decrease in biodiversity. These shortcomings prompted the policymakers and researchers to focus on the use of alternative environmentally friendly management strategies to manage target pest species. Among alternative methods, plant-based pesticides and naturally occurring bio-control agents attained considerable importance because of their high degree of host specificity and high searching ability, respectively. The use of biopesticides to manage other mite species have been described in numerous investigations [6-12]. The spores of the strain of M. anisopliae selected for the current study had shown virulence against various pest species including Ocinara varians Walker [13], Coptotermes formosanus Shiraki [14,15], and O. afrasiaticus (McGregor) [16]. On the other hand, plant secondary metabolite, 1-Chlorooctadecane selected for the current study is a promising pest management candidate known to extract from numerous plant species including Albertisia papuana Becc [17], Syzygium cumini (L.) [18], Arisaema amurense Maxim [19], and fungus, Trichoderma harzianum [20]. 1-Chlorooctadecane $\left(\mathrm{CH}_{3}\right.$ $\left(\mathrm{CH}_{2}\right) 16 \mathrm{CH}_{2} \mathrm{Cl}$ ) was screened from our preliminary study on the chemical fractionation of Cucumis sativus that showed toxicity against date palm dust mites. The successful implementation of these agents can yield numerous benefits such as no side effects, cost-effectiveness, reduced reliance on conventional pesticides, minimum environmental disturbance and self-perpetuation. However, few studies have explored the potential of biopesticides to manage O. afrasiaticus populations [16,21-23]. The current study is primarily aimed to fully exploit for the first time the biocontrol potential of plant secondary metabolite, 1-Chlorooctadecane and pathogenic fungus, M. anisopliae EBCL 02049 by a series of experimentation including compatibility assays, concentration-mortality response bioassays, physiological enzymatic regulations, and joint toxicity index analysis in order to facilitate a knowledge-based eco-friendly date palm dust mites management approach.

\section{Results}

\subsection{Compatibility Bioassays}

The spores of $M$. anisopliae EBCL 02049 were found to be compatible because they showed a very high value of biological index (BI > 82), against all the tested concentrations of 1-Chlorooctadecane. However, we found concentration-dependent inversely proportional relationship between concentrations of 1-Chlorooctadecane and BI. All the tested concentrations of 1-Chlorooctadecane revealed significant differences in the vegetative growth $(F=3.32 ; d f=5,54$; $p=0.011)$, and sporulation $(F=3.00 ; d f=5,54 ; p=0.019)$ of $M$. anisopliae EBCL 02049. We recorded a similar concentration-dependent response. The lower concentrations failed to inhibit the fungal 
growth resulting in significantly wider vegetative mycelial growth and high sporulation of $M$. anisopliae EBCL 02049 (Table 1). On the other hand, spores germination of M. anisopliae EBCL 02049 against all the tested concentrations revealed non-significant interaction $(F=0.93 ; d f=5,54 ; p=0.468)$, and resulting very high percent germination $(>96 \%)$.

Table 1. Compatibility of 1-Chlorooctadecane with the spores of M. anisopliae.

\begin{tabular}{cccccc}
\hline Treatments & $\begin{array}{c}\text { Vegetative } \\
\text { Growth }(\mathbf{m m})^{\mathbf{1}}\end{array}$ & $\begin{array}{c}\text { Germination } \\
\mathbf{( \% )}^{\mathbf{1}}\end{array}$ & $\begin{array}{c}\text { Sporulation } \\
\left(\times \mathbf{1 0}^{\mathbf{6}} \mathbf{S p o r e s}_{\mathbf{m L})} \mathbf{1}\right.\end{array}$ & $\begin{array}{c}\text { Biological } \\
\text { Index }\end{array}$ & Classification $^{\mathbf{2}}$ \\
\hline Control & $85.70 \pm 2.25^{\mathrm{a}}$ & $98.70 \pm 0.79^{\mathrm{a}}$ & $7.80 \pm 0.63^{\mathrm{a}}$ & - & - \\
$0.8 \mathrm{mg} / \mathrm{mL}$ & $84.40 \pm 2.28^{\mathrm{ab}}$ & $98.20 \pm 0.99^{\mathrm{a}}$ & $7.30 \pm 0.60^{\mathrm{ab}}$ & 96.48 & Compatible \\
$1.6 \mathrm{mg} / \mathrm{mL}$ & $84.20 \pm 21.69^{\mathrm{ab}}$ & $97.90 \pm 1.03^{\mathrm{a}}$ & $6.40 \pm 0.54^{\mathrm{abc}}$ & 91.38 & Compatible \\
$2.4 \mathrm{mg} / \mathrm{mL}$ & $79.30 \pm 1.82^{\mathrm{bc}}$ & $97.30 \pm 0.71^{\mathrm{a}}$ & $6.20 \pm 0.61^{\mathrm{bc}}$ & 87.53 & Compatible \\
$3.2 \mathrm{mg} / \mathrm{mL}$ & $78.10 \pm 2.11^{\mathrm{c}}$ & $96.70 \pm 0.93^{\mathrm{a}}$ & $5.60 \pm 0.40^{\mathrm{c}}$ & 83.50 & Compatible \\
$4.0 \mathrm{mg} / \mathrm{mL}$ & $77.70 \pm 1.54^{\mathrm{c}}$ & $97.20 \pm 0.81^{\mathrm{a}}$ & $5.50 \pm 0.34^{\mathrm{c}}$ & 82.78 & Compatible \\
\hline
\end{tabular}

${ }^{1}$ Numerical values (means \pm SE) are the means of ten replicates. Different lower-case letter(s) superscript followed by means \pm SE within a column are significantly different (Fisher's LSD test; $\alpha=0.05$ ). ${ }^{2}$ Biological index-based classification criterion: BI more than 66 classified as Compatible, BI between 42 to 66 classified as Moderately toxic; BI less than or equal to 42 classified as Toxic interaction.

\subsection{Screening Biossays}

Date palm dust mites exposed with different concentrations of 1-Chlorooctadecane, and M. anisopliae EBCL 02049 spores alone or in different proportions revealed concentration-dependent mortality response (Figure 1). The sole application of M. anisopliae EBCL 02049 spore suspensions at different concentrations exhibited the lowest mortality response resulting the greater value for $\mathrm{LC}_{50}$ $(21.66 \mathrm{mg} / \mathrm{mL})$. However, all the tested concentrations of the spores $(F=920.10 ; d f=4,48 ; p<0.0001)$, at different time intervals $(F=194.27 ; d f=2,48 ;<0.0001)$, and their interaction $(F=121.91 ; d f=8,48$; $<0.0001$ ), revealed significant differences in the mortality of date palm dust mites (Figure 1b). On the other hand, sole application of 1-Chlorooctadecane has shown comparatively lower value for $\mathrm{LC}_{50}$ $(3.42 \mathrm{mg} / \mathrm{mL})$. The mortality response of date palm dust mites recorded at different time intervals $(F=138.93 ; d f=2,48 ;<0.0001)$, after different concentrations of 1-Chlorooctadecane $(F=1181.20$; $d f=4,48 ; p<0.0001)$, and their interaction $(F=140.63 ; d f=8,48 ;<0.0001)$ exhibited significant differences (Figure 1a).

Different bioassay schemes designed to screen the most potent interaction revealed variable interaction response in terms of the mortality of second nymphal stage of date palm dust mites. The combination of 1-Chlorooctadecane with spores mentioned in Table 2 as Scheme I (20\% 1-Chlorooctadecane: $80 \%$ Spores) led to an antagonistic interaction by revealing the least joint toxicity (joint toxicity $=47$ ). However, mortality response of date palm dust mites exposed to different concentrations of these combinations $(F=819.31 ; d f=4,48 ; p<0.0001)$, recorded at different time intervals $(F=179.31 ; d f=2,48 ;<0.0001)$, and their interaction $(F=75.94 ; d f=8,48 ;<0.0001)$ revealed significant differences (Figure 1I). However, the combination Scheme II in which different concentrations were prepared to finalize the final concentrations with combination strength of $40 \%$ 1-Chlorooctadecane: $60 \%$ Spores led to a synergistic interaction (joint toxicity $=112$ ). The mortality responses of date palm dust mites displayed in the Figure 1II revealed significant differences at different concentrations $(F=439.53 ; d f=4,48 ; p<0.0001)$, time intervals $(F=265.19 ; d f=2,48 ;<0.0001)$, and their interaction $(F=26.81 ; d f=8,48 ;<0.0001)$.

The combined application of 1-Chlorooctadecane and spores (Scheme III: 60\% 1-Chlorooctadecane: $40 \%$ Spores) imparted significant differences in the mortality of date palm dust mites against different concentrations ( $F=1345.03 ; d f=4,48 ; p<0.0001)$, time intervals $(F=365.97 ; d f=2,48 ;<0.0001)$, and their interaction $(F=77.38 ; d f=8,48 ;<0.0001)$ as shown in Figure 1III. On the other hand, joint toxicity recorded as a result of Scheme III combinations exhibited bit higher value (joint toxicity $=$ 289 ) as shown in Table 2 . Similarly, strong synergistic interaction (joint toxicity $=713$ ) was recorded 
from the Scheme IV bioassays in which different concentrations were prepared to finalize the final concentrations with combination strength of 80\% 1-Chlorooctadecane: 20\% Spores (Table 2). The mortality response of date palm dust mites exposed to different concentrations of these combinations $(F=1275.55 ; d f=4,48 ; p<0.0001)$, recorded at different time intervals $(F=394.27 ; d f=2,48 ;<0.0001)$, and their interaction $(F=112.46 ; d f=8,48 ;<0.0001)$, revealed significant differences (Figure 1IV).

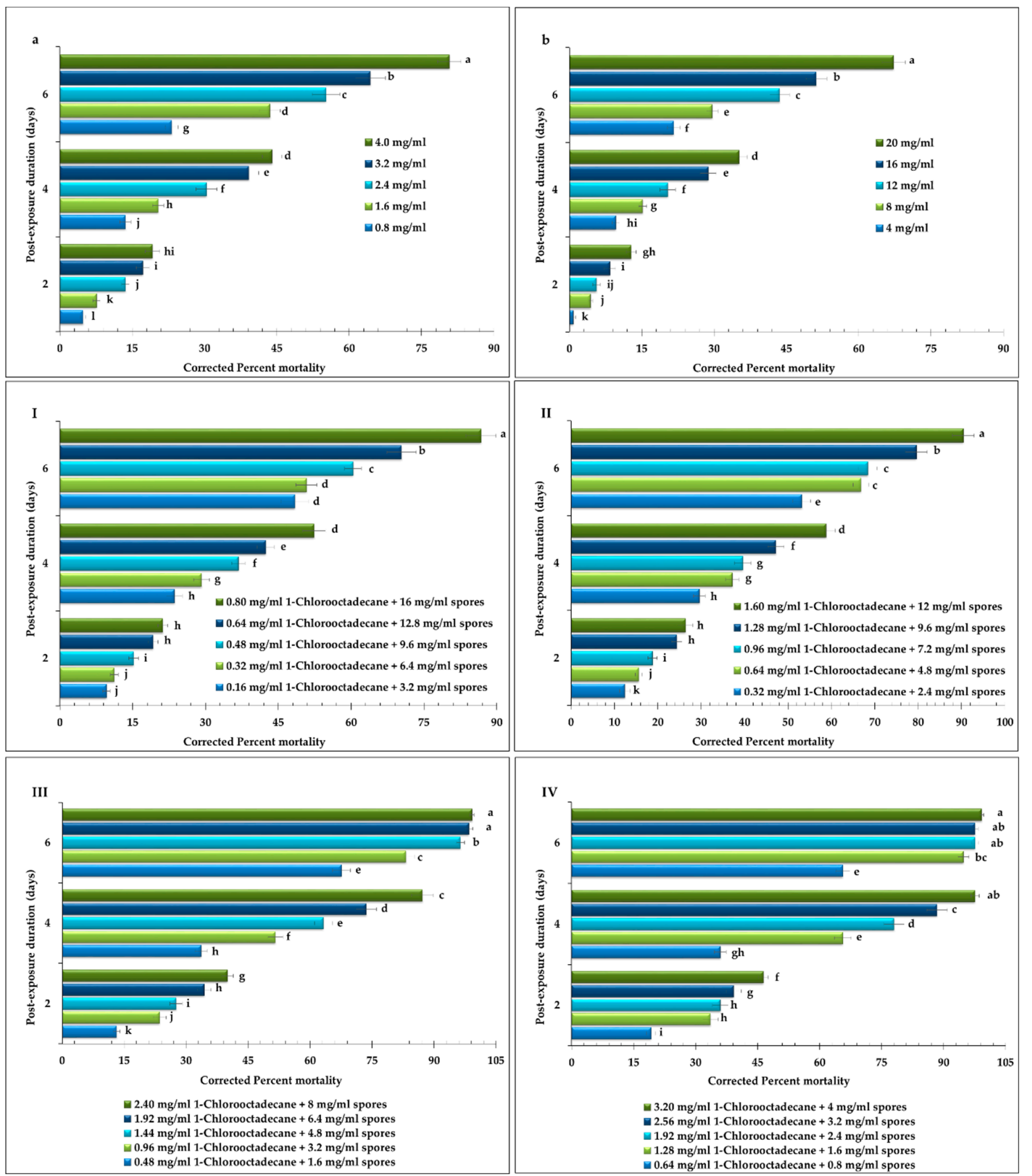

Figure 1. Impact of various concentrations of (a) 1-Chlorooctadecane; (b) suspensions of $M$. anisopliae; and their various interactions (I) 20\% 1-Chlorooctadecane: 80\% Spores; (II) 40\% 1-Chlorooctadecane: 80\% Spores; (III) 60\% 1-Chlorooctadecane: 40\% Spores; (IV) 80\% 1-Chlorooctadecane: 20\% Spores; on the mortality of second nymphal stage date palm dust mites. The comparisons of mortalities of $O$. afrasiaticus among different treatments were evaluated by repeated measures ANOVA (Fisher's LSD test; $\alpha=0.05)$. 
Table 2. Toxicity of the interactions between 1-Chlorooctadecane and the spores of M. anisopliae against date palm dust mites.

\begin{tabular}{|c|c|c|c|}
\hline Combinations & $\mathrm{LC}_{50}(\mathrm{mg} / \mathrm{mL})$ & Joint Toxicity & Interaction ${ }^{1}$ \\
\hline \multicolumn{4}{|c|}{$\begin{array}{l}\text { Scheme I: } 20 \% \text { 1-Chlorooctadecane: } 80 \% \text { Spores } \\
\end{array}$} \\
\hline \multicolumn{4}{|c|}{$0.16 \mathrm{mg} / \mathrm{mL}$ 1-Chlorooctadecane $+3.2 \mathrm{mg} / \mathrm{mL}$ spores } \\
\hline \multicolumn{4}{|l|}{$0.32 \mathrm{mg} / \mathrm{mL}$ 1-Chlorooctadecane $+6.4 \mathrm{mg} / \mathrm{mL}$ spores } \\
\hline $0.48 \mathrm{mg} / \mathrm{mL} 1$-Chlorooctadecane $+9.6 \mathrm{mg} / \mathrm{mL}$ spores & $8.75(7.10-10.75)$ & 47 & Antagonistic \\
\hline \multicolumn{4}{|l|}{$0.64 \mathrm{mg} / \mathrm{mL}$ 1-Chlorooctadecane $+12.8 \mathrm{mg} / \mathrm{mL}$ spores } \\
\hline \multicolumn{4}{|c|}{$0.80 \mathrm{mg} / \mathrm{mL} 1$-Chlorooctadecane $+16.0 \mathrm{mg} / \mathrm{mL}$ spores } \\
\hline \multicolumn{4}{|c|}{ Scheme II: $40 \%$ 1-Chlorooctadecane: $60 \%$ Spores } \\
\hline \multicolumn{4}{|c|}{$0.32 \mathrm{mg} / \mathrm{mL}$ 1-Chlorooctadecane $+2.4 \mathrm{mg} / \mathrm{mL}$ spores } \\
\hline \multicolumn{4}{|l|}{$0.64 \mathrm{mg} / \mathrm{mL}$ 1-Chlorooctadecane $+4.8 \mathrm{mg} / \mathrm{mL}$ spores } \\
\hline $0.96 \mathrm{mg} / \mathrm{mL}$ 1-Chlorooctadecane $+7.2 \mathrm{mg} / \mathrm{mL}$ spores & $4.62(3.41-5.65)$ & 112 & Synergistic \\
\hline \multicolumn{4}{|l|}{$1.28 \mathrm{mg} / \mathrm{mL} 1$-Chlorooctadecane $+9.6 \mathrm{mg} / \mathrm{mL}$ spores } \\
\hline \multicolumn{4}{|c|}{$1.60 \mathrm{mg} / \mathrm{mL} 1$-Chlorooctadecane $+12 \mathrm{mg} / \mathrm{mL}$ spores } \\
\hline \multicolumn{4}{|c|}{ Scheme III: $60 \%$ 1-Chlorooctadecane: $40 \%$ Spores } \\
\hline \multicolumn{4}{|c|}{$0.48 \mathrm{mg} / \mathrm{mL}$ 1-Chlorooctadecane $+1.6 \mathrm{mg} / \mathrm{mL}$ spores } \\
\hline \multicolumn{4}{|l|}{$0.96 \mathrm{mg} / \mathrm{mL}$ 1-Chlorooctadecane $+3.2 \mathrm{mg} / \mathrm{mL}$ spores } \\
\hline $1.44 \mathrm{mg} / \mathrm{mL}$ 1-Chlorooctadecane $+4.8 \mathrm{mg} / \mathrm{mL}$ spores & $2.39(2.00-2.72)$ & 289 & Synergistic \\
\hline \multicolumn{4}{|l|}{$1.92 \mathrm{mg} / \mathrm{mL}$ 1-Chlorooctadecane $+6.4 \mathrm{mg} / \mathrm{mL}$ spores } \\
\hline \multicolumn{4}{|c|}{$2.40 \mathrm{mg} / \mathrm{mL}$ 1-Chlorooctadecane $+8.0 \mathrm{mg} / \mathrm{mL}$ spores } \\
\hline \multicolumn{4}{|c|}{ Scheme IV: $80 \%$ 1-Chlorooctadecane: $20 \%$ Spores } \\
\hline \multirow{2}{*}{\multicolumn{4}{|c|}{$\begin{array}{l}0.64 \mathrm{mg} / \mathrm{mL} \text { 1-Chlorooctadecane }+0.8 \mathrm{mg} / \mathrm{mL} \text { spores } \\
1.28 \mathrm{mg} / \mathrm{mL} \text { 1-Chlorooctadecane }+1.6 \mathrm{mg} / \mathrm{mL} \text { spores }\end{array}$}} \\
\hline & & & \\
\hline $1.92 \mathrm{mg} / \mathrm{mL}$ 1-Chlorooctadecane $+2.4 \mathrm{mg} / \mathrm{mL}$ spores & $1.47(1.24-1.67)$ & 713 & Synergistic \\
\hline $2.56 \mathrm{mg} / \mathrm{mL}$ 1-Chlorooctadecane $+3.2 \mathrm{mg} / \mathrm{mL}$ spores & & & \\
\hline $3.20 \mathrm{mg} / \mathrm{mL}$ 1-Chlorooctadecane $+4.0 \mathrm{mg} / \mathrm{mL}$ spores & & & \\
\hline
\end{tabular}

${ }^{1}$ Interaction criterion: Joint toxicity less than 100 is classified as Antagonistic. 


\subsection{Host Antioxidant Defense Response}

The exposure of different treatments sole or in different combination schemes produced various levels of catalase (CAT), and superoxide dismutase (SOD) activities relative to control treatment. The relative CAT activities of $O$. afrasiaticus analyzed after $24 \mathrm{~h}$ post-exposure revealed significant differences among different treatments $(F=256.60 ; d f=5,120 ; p<0.0001)$, concentrations $(F=228.63 ; d f=4,120$; $p<0.0001)$, and their interaction $(F=2.35 ; d f=20,120 ; p=0.0031)$. Overall, the most potent treatment especially the treatments from the higher concentrations of Scheme IV (80\% 1-Chlorooctadecane: $20 \%$ Spores) tremendously induced the enzymatic activities of CAT, and remained statistically at the highest level. On the other hand, the lowest concentration of 1-Chlorooctadecane $(0.8 \mathrm{mg} / \mathrm{mL})$ after $24 \mathrm{~h}$ of post-exposure induced the lowest CAT activities among date palm dust mites (Table 3). In contrast, $0.16 \mathrm{mg} / \mathrm{mL}$ of 1 -Chlorooctadecane $+3.2 \mathrm{mg} / \mathrm{mL}$ of spores induced the lowest CAT activities among date palm dust mites exposed for $72 \mathrm{~h}$. However, all the treatments $(F=208.53 ; d f=5,120$; $p<0.0001)$, with different concentrations $(F=464.81 ; d f=4,120 ; p<0.0001)$, and their interaction $(F=103.21 ; d f=20,120 ; p<0.0001)$, revealed significant differences in the relative CAT activities from $72 \mathrm{~h}$ post-exposure date palm dust mites (Table 3). In addition, similar to $24 \mathrm{~h}$ post-exposure, date palm dust mites exposed for $72 \mathrm{~h}$ also tremendously induced CAT activities in response to the most potent treatment $(3.20 \mathrm{mg} / \mathrm{mL} 1$-Chlorooctadecane $+4.0 \mathrm{mg} / \mathrm{mL}$ spores). Unlike to relative CAT activities recorded $72 \mathrm{~h}$ post-exposure, the most potent treatments including the highest concentrations of Scheme IV (80\% 1-Chlorooctadecane: $20 \%$ Spores) failed to induce the enzymatic activities of CAT after $120 \mathrm{~h}$ post-exposure, and remained statistically at the lowest level (Table 3). At this time interval, M. anisopliae EBCL 02049 spores at a concentration of $8 \mathrm{mg} / \mathrm{mL}$ induced the highest level of relative CAT activities of infected date palm dust mites, and remained statistically at the highest level. Overall, relative CAT activities recorded after $120 \mathrm{~h}$ showed significant differences against different treatments $(F=3621.36 ; d f=5,120 ; p<0.0001)$, concentrations $(F=1536.63 ; d f=4,120 ; p<0.0001)$, and their interaction $(F=105.06 ; d f=20,120 ; p<0.0001)$.

Relative enzymatic activities of SOD recorded from date palm dust mites after $24 \mathrm{~h}$ of exposure against different treatments $(F=331.28 ; d f=5,120 ; p<0.0001)$, concentrations $(F=398.14 ; d f=4,120$; $p<0.0001)$, and their interaction $(F=5.44 ; d f=20,120 ; p<0.0001)$, revealed significant differences (Table 4$)$. The infection of the least potent treatment $(4 \mathrm{mg} / \mathrm{mL}$ spores of $M$. anisopliae EBCL02049) failed to induce SOD activities and remained at the lowest level. On the other hand, $3.20 \mathrm{mg} / \mathrm{mL}$ 1-Chlorooctadecane $+4.0 \mathrm{mg} / \mathrm{mL}$ spores triggered the SOD activities. This trend continues to increase until $72 \mathrm{~h}$ post-exposure, and resulted the highest relative SOD activities from this potent treatment. Furthermore, significant differences in relative SOD activities calculated against different treatments $(F=340.46 ; d f=5,120 ; p<0.0001)$, concentrations $(F=759.10 ; d f=4,120 ; p<0.0001)$, and their interaction $(F=164.03 ; d f=20,120 ; p<0.0001)$, were recorded in this study. 
Table 3. Relative catalase (CAT) activities of date palm dust mites fed for different time intervals on date palm leaf-discs treated solely and in different combinations of 1-Chlorooctadecane with M. anisopliae spores.

\begin{tabular}{|c|c|c|c|}
\hline \multirow{2}{*}{ Treatments } & \multicolumn{3}{|c|}{ Post-Exposure Duration } \\
\hline & $24 h^{1}$ & $72 \mathrm{~h}^{1}$ & $120 \mathrm{~h}^{1}$ \\
\hline 1-Chlorooctadecane & $(\%)$ & $(\%)$ & $(\%)$ \\
\hline $0.8 \mathrm{mg} / \mathrm{mL}$ & $13.35 \pm 0.55^{\mathrm{P}}$ & $32.18 \pm 0.34^{\mathrm{m}}$ & $46.03 \pm 0.80 \mathrm{~g}$ \\
\hline $1.6 \mathrm{mg} / \mathrm{mL}$ & $20.32 \pm 1.02^{\mathrm{lm}}$ & $35.56 \pm 0.49^{1}$ & $39.59 \pm 0.88^{\mathrm{i}}$ \\
\hline $2.4 \mathrm{mg} / \mathrm{mL}$ & $21.17 \pm 1.03^{\mathrm{kl}}$ & $40.09 \pm 0.69^{\mathrm{ij}}$ & $23.27 \pm 0.44^{1}$ \\
\hline $3.2 \mathrm{mg} / \mathrm{mL}$ & $24.38 \pm 1.18^{\mathrm{hi}}$ & $46.16 \pm 0.97 \mathrm{~g}$ & $17.13 \pm 0.34^{\circ \mathrm{op}}$ \\
\hline $4.0 \mathrm{mg} / \mathrm{mL}$ & $27.14 \pm 1.32^{\mathrm{fg}}$ & $52.86 \pm 0.92^{\mathrm{d}}$ & $15.14 \pm 0.25 \mathrm{q}$ \\
\hline \multicolumn{4}{|l|}{ Scheme I: $20 \%$ 1-Chlorooctadecane: $80 \%$ Spores } \\
\hline $0.16 \mathrm{mg} / \mathrm{mL}$ 1-Chlorooctadecane $+3.2 \mathrm{mg} / \mathrm{mL}$ spores & $15.04 \pm 0.21^{\mathrm{op}}$ & $31.52 \pm 0.37^{\mathrm{m}}$ & $60.78 \pm 0.94^{\mathrm{b}}$ \\
\hline $0.32 \mathrm{mg} / \mathrm{mL}$ 1-Chlorooctadecane $+6.4 \mathrm{mg} / \mathrm{mL}$ spores & $16.38 \pm 0.24^{\text {no }}$ & $39.64 \pm 0.59 j$ & $53.88 \pm 0.98^{\mathrm{e}}$ \\
\hline $0.48 \mathrm{mg} / \mathrm{mL}$ 1-Chlorooctadecane $+9.6 \mathrm{mg} / \mathrm{mL}$ spores & $20.22 \pm 0.54^{\mathrm{lm}}$ & $44.17 \pm 0.72^{g h}$ & $56.42 \pm 0.93^{\mathrm{d}}$ \\
\hline $0.64 \mathrm{mg} / \mathrm{mL}$ 1-Chlorooctadecane $+12.8 \mathrm{mg} / \mathrm{mL}$ spores & $21.19 \pm 0.78^{\mathrm{kl}}$ & $50.11 \pm 0.95^{\mathrm{ef}}$ & $52.78 \pm 0.95^{\mathrm{e}}$ \\
\hline $0.80 \mathrm{mg} / \mathrm{mL}$ 1-Chlorooctadecane $+16.0 \mathrm{mg} / \mathrm{mL}$ spores & $25.15 \pm 0.92^{\mathrm{ghi}}$ & $43.11 \pm 0.84^{\mathrm{h}}$ & $41.46 \pm 0.76^{\mathrm{h}}$ \\
\hline \multicolumn{4}{|l|}{ Scheme II: $40 \%$ 1-Chlorooctadecane: $60 \%$ Spores } \\
\hline $0.32 \mathrm{mg} / \mathrm{mL}$ 1-Chlorooctadecane $+2.4 \mathrm{mg} / \mathrm{mL}$ spores & $20.32 \pm 0.31^{\mathrm{lm}}$ & $35.30 \pm 0.55^{1}$ & $40.03 \pm 0.64^{\mathrm{hi}}$ \\
\hline $0.64 \mathrm{mg} / \mathrm{mL}$ 1-Chlorooctadecane $+4.8 \mathrm{mg} / \mathrm{mL}$ spores & $22.06 \pm 0.70^{\mathrm{kl}}$ & $36.50 \pm 0.57^{1}$ & $29.74 \pm 0.55^{j}$ \\
\hline $0.96 \mathrm{mg} / \mathrm{mL}$ 1-Chlorooctadecane $+7.2 \mathrm{mg} / \mathrm{mL}$ spores & $27.15 \pm 0.72^{\mathrm{fg}}$ & $39.68 \pm 0.78^{j}$ & $24.09 \pm 0.50^{1}$ \\
\hline $1.28 \mathrm{mg} / \mathrm{mL}$ 1-Chlorooctadecane $+9.6 \mathrm{mg} / \mathrm{mL}$ spores & $30.01 \pm 0.90^{\mathrm{de}}$ & $48.45 \pm 0.91^{\mathrm{f}}$ & $20.10 \pm 0.24^{\mathrm{m}}$ \\
\hline $1.60 \mathrm{mg} / \mathrm{mL}$ 1-Chlorooctadecane $+12 \mathrm{mg} / \mathrm{mL}$ spores & $34.18 \pm 0.92^{\mathrm{bc}}$ & $52.93 \pm 0.93^{\mathrm{d}}$ & $15.20 \pm 0.20^{q}$ \\
\hline \multicolumn{4}{|l|}{ Scheme III: $60 \%$ 1-Chlorooctadecane: $40 \%$ Spores } \\
\hline $0.48 \mathrm{mg} / \mathrm{mL}$ 1-Chlorooctadecane $+1.6 \mathrm{mg} / \mathrm{mL}$ spores & $20.10 \pm 0.24^{\mathrm{lm}}$ & $35.69 \pm 0.46^{1}$ & $26.54 \pm 0.72^{\mathrm{k}}$ \\
\hline $0.96 \mathrm{mg} / \mathrm{mL}$ 1-Chlorooctadecane $+3.2 \mathrm{mg} / \mathrm{mL}$ spores & $24.19 \pm 0.60^{\text {hij }}$ & $37.43 \pm 0.56^{\mathrm{kl}}$ & $24.19 \pm 0.60^{1}$ \\
\hline $1.44 \mathrm{mg} / \mathrm{mL}$ 1-Chlorooctadecane $+4.8 \mathrm{mg} / \mathrm{mL}$ spores & $29.12 \pm 0.78^{\mathrm{ef}}$ & $42.02 \pm 0.70^{\mathrm{hi}}$ & $20.29 \pm 0.39^{\mathrm{m}}$ \\
\hline $1.92 \mathrm{mg} / \mathrm{mL}$ 1-Chlorooctadecane $+6.4 \mathrm{mg} / \mathrm{mL}$ spores & $32.22 \pm 0.87^{\mathrm{cd}}$ & $55.90 \pm 0.76^{\mathrm{c}}$ & $17.44 \pm 0.32^{\text {no }}$ \\
\hline $2.40 \mathrm{mg} / \mathrm{mL}$ 1-Chlorooctadecane $+8.0 \mathrm{mg} / \mathrm{mL}$ spores & $33.22 \pm 0.90^{\mathrm{bc}}$ & $63.85 \pm 0.93^{\mathrm{b}}$ & $09.79 \pm 0.26^{\mathrm{r}}$ \\
\hline \multicolumn{4}{|l|}{ Scheme IV: $80 \%$ 1-Chlorooctadecane: $20 \%$ Spores } \\
\hline $0.64 \mathrm{mg} / \mathrm{mL}$ 1-Chlorooctadecane $+0.8 \mathrm{mg} / \mathrm{mL}$ spores & $23.08 \pm 0.57^{\mathrm{ijk}}$ & $38.92 \pm 0.57 \mathrm{j}^{\mathrm{k}}$ & $15.58 \pm 0.28^{\mathrm{pq}}$ \\
\hline $1.28 \mathrm{mg} / \mathrm{mL}$ 1-Chlorooctadecane $+1.6 \mathrm{mg} / \mathrm{mL}$ spores & $29.37 \pm 0.64^{\mathrm{ef}}$ & $43.35 \pm 0.69^{\mathrm{h}}$ & $09.67 \pm 0.25^{\mathrm{r}}$ \\
\hline $1.92 \mathrm{mg} / \mathrm{mL}$ 1-Chlorooctadecane $+2.4 \mathrm{mg} / \mathrm{mL}$ spores & $33.02 \pm 0.81^{\mathrm{bc}}$ & $50.16 \pm 0.79^{\mathrm{ef}}$ & $03.58 \pm 0.24^{\mathrm{s}}$ \\
\hline $2.56 \mathrm{mg} / \mathrm{mL}$ 1-Chlorooctadecane $+3.2 \mathrm{mg} / \mathrm{mL}$ spores & $35.12 \pm 0.93^{\mathrm{b}}$ & $63.96 \pm 0.91^{\mathrm{b}}$ & $01.79 \pm 0.15^{\mathrm{t}}$ \\
\hline $3.20 \mathrm{mg} / \mathrm{mL}$ 1-Chlorooctadecane $+4.0 \mathrm{mg} / \mathrm{mL}$ spores & $39.32 \pm 1.22^{\mathrm{a}}$ & $71.64 \pm 1.05^{\mathrm{a}}$ & $01.24 \pm 0.13^{\mathrm{t}}$ \\
\hline \multicolumn{4}{|l|}{$\begin{array}{l}\text { M. anisopliae EBCL02049 spores } \\
\text { (n) }\end{array}$} \\
\hline $4 \mathrm{mg} / \mathrm{mL}$ & $15.34 \pm 0.43^{\circ \mathrm{p}}$ & $49.13 \pm 0.81^{\mathrm{f}}$ & $58.19 \pm 0.81^{\mathrm{c}}$ \\
\hline $8 \mathrm{mg} / \mathrm{mL}$ & $18.27 \pm 0.56^{\mathrm{mn}}$ & $57.16 \pm 0.98^{c}$ & $63.83 \pm 0.77^{\mathrm{a}}$ \\
\hline $12 \mathrm{mg} / \mathrm{mL}$ & $21.08 \pm 0.78^{\mathrm{kl}}$ & $51.35 \pm 0.97 \mathrm{de}$ & $48.38 \pm 0.97^{\mathrm{f}}$ \\
\hline $16 \mathrm{mg} / \mathrm{mL}$ & $23.26 \pm 0.88^{\mathrm{ijk}}$ & $43.67 \pm 0.93^{\mathrm{h}}$ & $28.07 \pm 0.62^{\mathrm{j}}$ \\
\hline $20 \mathrm{mg} / \mathrm{mL}$ & $26.17 \pm 0.91^{\mathrm{gh}}$ & $36.26 \pm 0.68^{1}$ & $19.13 \pm 0.60^{\mathrm{mn}}$ \\
\hline
\end{tabular}

${ }^{1}$ Numerical values (means $\pm \mathrm{SE}$ ) are the means of five replicates. Different lower-case letter(s) superscript followed by means \pm SE of relative CAT activities of $O$. afrasiaticus within the column are significantly different (Fisher's LSD test; $\alpha=0.05$ ). 
Table 4. Relative superoxide dismutase (SOD) activities of date palm dust mites fed for different time intervals on date palm leaf-discs treated solely and in different combinations of 1-Chlorooctadecane with M. anisopliae spores.

\begin{tabular}{|c|c|c|c|}
\hline \multirow{2}{*}{ Treatments } & \multicolumn{3}{|c|}{ Post-Exposure Duration } \\
\hline & $24 h^{1}$ & $72 \mathrm{~h}^{1}$ & $120 \mathrm{~h}^{1}$ \\
\hline 1-Chlorooctadecane & $(\%)$ & $(\%)$ & $(\%)$ \\
\hline $0.8 \mathrm{mg} / \mathrm{mL}$ & $12.89 \pm 0.53^{\mathrm{kl}}$ & $30.38 \pm 0.85^{q}$ & $66.27 \pm 1.19^{\mathrm{bc}}$ \\
\hline $1.6 \mathrm{mg} / \mathrm{mL}$ & $20.42 \pm 0.78^{\mathrm{h}}$ & $36.15 \pm 0.90^{\circ} \mathrm{p}$ & $57.13 \pm 1.21^{\mathrm{d}}$ \\
\hline $2.4 \mathrm{mg} / \mathrm{mL}$ & $21.20 \pm 0.87^{\mathrm{h}}$ & $43.85 \pm 0.93^{1}$ & $37.31 \pm 0.65^{\mathrm{h}}$ \\
\hline $3.2 \mathrm{mg} / \mathrm{mL}$ & $26.87 \pm 1.05^{\mathrm{d}}$ & $54.04 \pm 1.27 \mathrm{~g}$ & $31.36 \pm 0.45^{\mathrm{ij}}$ \\
\hline $4.0 \mathrm{mg} / \mathrm{mL}$ & $29.65 \pm 1.29^{c}$ & $66.10 \pm 1.35^{\mathrm{c}}$ & $19.77 \pm 0.36^{1 \mathrm{~m}}$ \\
\hline \multicolumn{4}{|l|}{ Scheme I: 20\% 1-Chlorooctadecane: $80 \%$ Spores } \\
\hline $0.16 \mathrm{mg} / \mathrm{mL}$ 1-Chlorooctadecane $+3.2 \mathrm{mg} / \mathrm{mL}$ spores & $11.17 \pm 0.27^{1}$ & $53.13 \pm 0.97^{\mathrm{gh}}$ & $76.13 \pm 1.01^{\mathrm{a}}$ \\
\hline $0.32 \mathrm{mg} / \mathrm{mL}$ 1-Chlorooctadecane $+6.4 \mathrm{mg} / \mathrm{mL}$ spores & $13.33 \pm 0.42^{\mathrm{k}}$ & $61.18 \pm 0.99^{\mathrm{e}}$ & $68.07 \pm 0.98^{\mathrm{b}}$ \\
\hline $0.48 \mathrm{mg} / \mathrm{mL}$ 1-Chlorooctadecane $+9.6 \mathrm{mg} / \mathrm{mL}$ spores & $18.13 \pm 0.50^{j}$ & $52.54 \pm 1.04^{\mathrm{gh}}$ & $57.30 \pm 1.11^{\mathrm{d}}$ \\
\hline $0.64 \mathrm{mg} / \mathrm{mL}$ 1-Chlorooctadecane $+12.8 \mathrm{mg} / \mathrm{mL}$ spores & $20.11 \pm 0.76^{\mathrm{hi}}$ & $46.57 \pm 0.95^{j}$ & $40.43 \pm 0.69 \mathrm{~g}$ \\
\hline $0.80 \mathrm{mg} / \mathrm{mL}$ 1-Chlorooctadecane $+16.0 \mathrm{mg} / \mathrm{mL}$ spores & $21.32 \pm 0.85^{\text {gh }}$ & $29.46 \pm 0.50^{q}$ & $25.30 \pm 0.58^{\mathrm{k}}$ \\
\hline \multicolumn{4}{|l|}{ Scheme II: $40 \%$ 1-Chlorooctadecane: $60 \%$ Spores } \\
\hline $0.32 \mathrm{mg} / \mathrm{mL}$ 1-Chlorooctadecane $+2.4 \mathrm{mg} / \mathrm{mL}$ spores & $14.17 \pm 0.61^{\mathrm{k}}$ & $37.27 \pm 0.61^{\circ}$ & $43.08 \pm 0.82^{\mathrm{f}}$ \\
\hline $0.64 \mathrm{mg} / \mathrm{mL}$ 1-Chlorooctadecane $+4.8 \mathrm{mg} / \mathrm{mL}$ spores & $21.20 \pm 0.63^{\mathrm{h}}$ & $49.65 \pm 0.81^{\mathrm{i}}$ & $44.12 \pm 0.96^{\mathrm{f}}$ \\
\hline $0.96 \mathrm{mg} / \mathrm{mL}$ 1-Chlorooctadecane $+7.2 \mathrm{mg} / \mathrm{mL}$ spores & $25.42 \pm 0.91^{\text {de }}$ & $58.40 \pm 1.18^{\mathrm{f}}$ & $39.80 \pm 0.88 \mathrm{~g}$ \\
\hline $1.28 \mathrm{mg} / \mathrm{mL}$ 1-Chlorooctadecane $+9.6 \mathrm{mg} / \mathrm{mL}$ spores & $27.07 \pm 0.93^{\mathrm{d}}$ & $61.44 \pm 1.20^{\mathrm{e}}$ & $25.46 \pm 0.51^{\mathrm{k}}$ \\
\hline $1.60 \mathrm{mg} / \mathrm{mL}$ 1-Chlorooctadecane $+12.0 \mathrm{mg} / \mathrm{mL}$ spores & $30.11 \pm 1.02 \mathrm{c}$ & $64.55 \pm 1.25^{\mathrm{cd}}$ & $21.42 \pm 0.50^{1}$ \\
\hline \multicolumn{4}{|l|}{ Scheme III: $60 \%$ 1-Chlorooctadecane: $40 \%$ Spores } \\
\hline $0.48 \mathrm{mg} / \mathrm{mL}$ 1-Chlorooctadecane $+1.6 \mathrm{mg} / \mathrm{mL}$ spores & $17.21 \pm 0.39 j$ & $41.14 \pm 0.77^{\mathrm{mn}}$ & $36.26 \pm 0.68^{\mathrm{h}}$ \\
\hline $0.96 \mathrm{mg} / \mathrm{mL}$ 1-Chlorooctadecane $+3.2 \mathrm{mg} / \mathrm{mL}$ spores & $23.10 \pm 0.65^{f g}$ & $44.39 \pm 0.85^{\mathrm{kl}}$ & $29.32 \pm 0.63^{j}$ \\
\hline $1.44 \mathrm{mg} / \mathrm{mL}$ 1-Chlorooctadecane $+4.8 \mathrm{mg} / \mathrm{mL}$ spores & $24.27 \pm 0.70^{\mathrm{ef}}$ & $54.25 \pm 1.03 \mathrm{~g}$ & $25.37 \pm 0.53^{\mathrm{k}}$ \\
\hline $1.92 \mathrm{mg} / \mathrm{mL}$ 1-Chlorooctadecane $+6.4 \mathrm{mg} / \mathrm{mL}$ spores & $29.22 \pm 0.95^{c}$ & $63.76 \pm 1.24^{\mathrm{d}}$ & $20.54 \pm 0.43^{1}$ \\
\hline $2.40 \mathrm{mg} / \mathrm{mL} \mathrm{1-Chlorooctadecane}+8.0 \mathrm{mg} / \mathrm{mL}$ spores & $34.20 \pm 1.02^{\mathrm{a}}$ & $72.26 \pm 1.25^{\mathrm{b}}$ & $16.28 \pm 0.25^{\mathrm{n}}$ \\
\hline \multicolumn{4}{|l|}{ Scheme IV: $80 \%$ 1-Chlorooctadecane: $20 \%$ Spores } \\
\hline $0.64 \mathrm{mg} / \mathrm{mL}$ 1-Chlorooctadecane $+0.8 \mathrm{mg} / \mathrm{mL}$ spores & $23.38 \pm 0.52^{\mathrm{f}}$ & $43.15 \pm 0.81^{1 \mathrm{~m}}$ & $32.41 \pm 0.52^{\mathrm{i}}$ \\
\hline $1.28 \mathrm{mg} / \mathrm{mL}$ 1-Chlorooctadecane $+1.6 \mathrm{mg} / \mathrm{mL}$ spores & $25.32 \pm 0.70^{\text {de }}$ & $53.19 \pm 0.91 \mathrm{gh}^{\mathrm{gh}}$ & $26.42 \pm 0.43^{\mathrm{k}}$ \\
\hline $1.92 \mathrm{mg} / \mathrm{mL}$ 1-Chlorooctadecane $+2.4 \mathrm{mg} / \mathrm{mL}$ spores & $26.19 \pm 0.98^{\mathrm{d}}$ & $60.45 \pm 0.99^{\text {ef }}$ & $21.24 \pm 0.37^{1}$ \\
\hline $2.56 \mathrm{mg} / \mathrm{mL}$ 1-Chlorooctadecane $+3.2 \mathrm{mg} / \mathrm{mL}$ spores & $32.34 \pm 1.12^{\mathrm{b}}$ & $66.23 \pm 1.10^{c}$ & $18.03 \pm 0.52^{\mathrm{mn}}$ \\
\hline $3.20 \mathrm{mg} / \mathrm{mL}$ 1-Chlorooctadecane $+4.0 \mathrm{mg} / \mathrm{mL}$ spores & $35.41 \pm 1.18^{\mathrm{a}}$ & $81.28 \pm 1.26^{\mathrm{a}}$ & $11.80 \pm 0.37^{\circ}$ \\
\hline \multicolumn{4}{|l|}{ M. anisopliae EBCL02049 spores } \\
\hline $4 \mathrm{mg} / \mathrm{mL}$ & $09.28 \pm 0.34^{\mathrm{m}}$ & $34.20 \pm 0.79 \mathrm{P}$ & $56.53 \pm 0.93^{\mathrm{d}}$ \\
\hline $8 \mathrm{mg} / \mathrm{mL}$ & $17.11 \pm 0.48^{j}$ & $40.14 \pm 0.88^{\mathrm{n}}$ & $65.87 \pm 1.03^{\mathrm{c}}$ \\
\hline $12 \mathrm{mg} / \mathrm{mL}$ & $18.41 \pm 0.58^{\mathrm{ij}}$ & $46.43 \pm 0.92^{\mathrm{j}^{\mathrm{k}}}$ & $54.14 \pm 0.97^{\mathrm{e}}$ \\
\hline $16 \mathrm{mg} / \mathrm{mL}$ & $20.13 \pm 0.66^{\mathrm{hi}}$ & $51.24 \pm 0.98^{\mathrm{hi}}$ & $43.75 \pm 0.99^{f}$ \\
\hline $20 \mathrm{mg} / \mathrm{mL}$ & $23.16 \pm 0.89^{\mathrm{fg}}$ & $60.24 \pm 1.14^{\mathrm{ef}}$ & $25.46 \pm 0.72^{\mathrm{k}}$ \\
\hline
\end{tabular}

${ }^{1}$ Numerical values (means $\pm \mathrm{SE}$ ) are the means of five replicates. Different lower-case letter(s) superscript followed by means \pm SE of relative SOD activities of $O$. afrasiaticus within the column are significantly different (Fisher's LSD test; $\alpha=0.05$ ). 
Overall, the synergistic interaction combinations at their highest concentrations comparatively showed higher relative SOD activities compared with antagonistic interaction combinations (Table 4). On the contrary, relative SOD activities tend to decline after $120 \mathrm{~h}$ post-exposure among synergistic interactions compared with antagonistic interaction (Table 4). However, date palm dust mites showed significant differences among relative SOD activities in response to different treatments $(F=2230.32$; $d f=5,120 ; p<0.0001)$, concentrations $(F=1658.66 ; d f=4,120 ; p<0.0001)$, and their interaction $(F=71.62 ; d f=20,120 ; p<0.0001)$.

\section{Discussion}

Plant secondary metabolite 1-Chlorooctadecane improved the efficiency of M. anisopliae EBCL 02049 against date palm dust mites by reducing the killing time and increasing killing capacity. However, the extent of pathogenicity that was calculated through joint toxicity varied with the combining proportion of spores with 1-Chlorooctadecane. The simultaneous pairing of M. anisopliae EBCL 02049 spores with 1-Chlorooctadecane in a compatible manner as depicted from the biological index in the current study is ideally an interesting alternative approach against such pests, which are difficult to manage with a sole application of fungal spores.

The mortality data of the date palm dust mites in the present study indicated that the sole application of M. anisopliae EBCL 02049 produced relatively slow mortality and failed to kill all the tested populations. Slow mortality response of date palm dust mites is in line with what was previously reported against this strain of M. anisopliae EBCL 02049 [16]. These findings were further strengthened from previous studies on the virulence of entomopathogenic fungi against date palm dust mites [23,24]. The results of these studies revealed $100 \%$ mortality after 15-days post-exposure with $M$ anisopliae. Such a long time to impart mortality by the entomopathogenic fungi reported previously $[16,24,25]$, and shown in the current study in the form of low mortality even at the highest concentration $(20 \mathrm{mg}$ spores $/ \mathrm{mL}$ ) is demanding an alternative approach to overcome the difficulties in the way of eco-friendly pest management tactics.

Generally, a mixture of plant secondary metabolite with mycopathogen provides eco-friendly stable pest management system compared to environment-deteriorating chemical pesticides. However, the first step to develop a compatible mixture mainly depends on the interaction of these products that might lead to compatible or toxic interaction. The in vitro tests to evaluate the interaction of M. anisopliae EBCL 02049 spores with 1-Chlorooctadecane showed encouraging results in laboratory assays. Different concentrations of 1-Chlorooctadecane had little or no effect on the vegetative growth, germination, and sporulation of M. anisopliae EBCL 02049. These minor impacts of 1-Chlorooctadecane on growth parameters, which are mainly responsible for the propagation of fungus [26], lead to the value of the biological index under the range of compatible interaction. These compatible interactions at all tested doses of 1-Chlorooctadecane enabled us to support the choice for their simultaneous use in different proportions against date palm dust mites. The search on such a pairing remained a novel option against numerous pest species in order to circumvent slow and low mortality criticism of mycopathogen $[23,26-30]$.

It is well-known that both the fungi and plant secondary metabolites demonstrated an entirely different mode of actions to overcome the target host defense mechanisms to cause mortality. Fungal infection evades the host defense mechanism by triggering complex biochemical interactions in the form of series of events through cuticle adhesion, penetration, proliferation, and toxin production, which ultimately lead to the host mortality [29,31-33]. On the other hand, plant secondary metabolites are known antagonists that act by interfering with the signaling of the nervous and cellular systems to overcome the target host defense mechanism [34-38]. Therefore, the combined application is considered as a promising approach due to the differences in their mechanism of actions. Consequently, the mixture of fungal spores and toxin in a compatible manner were evaluated to develop synergistic interaction against various pest species $[23,26,28,39,40]$. The mixture of M. anisopliae EBCL 02049 and 1-Chlorooctadecane evaluated in the current study showed substantial synergistic interaction, 
which varies with their proportions. The cumulative corrected mortality of date palm dust mites infected with all the mixtures except $0.64 \mathrm{mg} / \mathrm{mL}$ 1-Chlorooctadecane $+0.8 \mathrm{mg} / \mathrm{mL}$ spores enlisted in Scheme IV (80\% 1-Chlorooctadecane: $20 \%$ Spores) was more than 95\%. Furthermore, LC $_{50}$ results (1.47 $\mathrm{mg} / \mathrm{mL}$ ) upon exposure with the range of mixtures from Scheme IV showed that these combinations more quickly killed the date palm dust mites compared to M. anisopliae EBCL 02049 spores and 1-Chlorooctadecane alone. These findings are in line with the previous study on the synergistic interaction of Beauveria bassiana spores with Phytol [23]. However, current results are more promising compared to a previous study (Joint toxicity $=691$ ), due to comparatively high toxicity (joint toxicity = 713). Furthermore, the findings of Zou et al., [40] on the joint action of fungal spores and toxin also strengthened our findings, which demonstrated that the synergistic interaction led to a quick mortality response of the combined treatment compared to their sole application. Similarly, the combined treatment enhanced the treatment effect revealed by Hernández et al., [28] against Tetranychus urticae further strengthened our findings. Their findings suggested that the compatibility of azadiractin with fungal spores against Tetranychus urticae revealed an additive effect, which contributes to improving their control by enhancing the treatment effect in the form of very high mortality. The outcome as a result of this study in the form of synergistic interaction undoubtedly will be useful for targeted management of date palm dust mites using natural products.

The application of the mixture of M. anisopliae EBCL 02049 spores and 1-Chlorooctadecane gave a varying antagonistic interaction especially from the bioassays of Scheme I (20\% 1-Chlorooctadecane: $80 \%$ Spores). There might be a number of explanations for this interaction. Akbar et al., [41], illustrated in their findings that putative antifungal activity of the toxin might contribute towards antagonistic interaction. The studies conducted against T. urticae population to find a compatible interaction of fungal spores and toxin revealed that adjustment of toxin concentration is very important to avoid antagonistic interaction [28]. These findings are in line with our results and enabled us to suggest that treatment efficacy should be regulated through optimization of pest management products to develop pest management synergistic interaction.

The antioxidant defense mechanism of the target host is promptly activated to remove the reactive oxygen species produced during stressful situations. The response of antioxidant enzymes calculated from the activities of SOD, and CAT, have shown treatment-specific and time-specific different patterns. The enzymatic activities of the front-line antioxidant enzyme, SOD against oxidative stress revealed distinct patterns at all the tested time intervals. The most potent treatment established in the current study significantly induced SOD activities until $72 \mathrm{~h}$ post-exposure. However, the lateral stage nutrient-deficient date palm dust mites failed to induce relative SOD activities and remained significantly at the lowest level. The induction of SOD activities under stressful situations has already been reported from several mite species. For instance, SOD activities in Tetranychus cinnabarinus fed on transgenic cassava lines resistant to this mite species were significantly induced compared with control mites [42]. The recent findings on the antioxidant response of date palm dust mites upon exposure with fungal infections with variable virulence range strengthened our findings by revealing similar patterns of SOD activities from potent treatments as depicted in the current study [24]. The initial increase in SOD activities established here and elsewhere mainly aimed to minimize the cellular damage by regulating the traffic of ROS generated as a result of oxidative stress by converting $R O S$ into $\mathrm{H}_{2} \mathrm{O}_{2}$. In the meanwhile, CAT is activated as described too in the current study, which detoxifies hydrogen peroxide by transforming into oxygen and water [43]. The least potent treatment in this study revealed high CAT activities over time for the safe removal of ROS corroborates with Hussain et al., [16]. On the other hand, enhanced CAT activities from the most potent treatment at the initial and middle staged infection, while negligible CAT activities from the late-staged nutrient-deficient infected mites are in agreement with previous studies conducted on different mite species on the exploration of antioxidant defense mechanism $[16,23,24,42]$. Such a pattern of defense enzymes may suggest that toxicity of the treatments regulates the host antioxidant enzyme activities. 


\section{Materials and Methods}

\subsection{Date Palm Dust Mites}

Populations of O. afrasiaticus for laboratory experimentation were directly collected from NCPD (National Center for Palms \& Dates), Al-Ahsa, Kingdom of Saudi Arabia. These populations were kept at $25 \pm 1{ }^{\circ} \mathrm{C} ; 62.5 \pm 12.5 \% \mathrm{RH}$, as mentioned in the previous study [24].

\subsection{M. anisopliae}

The strain EBCL 02049 of M. anisopliae isolated from Coptotermes formosanus Shiraki during 2002 from Jiangxi, China was grown on potato dextrose agar for twenty-four days under controlled conditions $\left(25 \pm 0.5^{\circ} \mathrm{C} ; 70 \pm 5 \% \mathrm{RH}\right)$. This particular strain was selected because their spores suspension has shown virulence against Ocinara varians Walker [13,44], Coptotermes formosanus Shiraki [14,15], and O. afrasiaticus (McGregor) [16]. The spore suspensions of required concentrations were prepared using 0.05\% Tween 80 (Sigma-Aldrich, London, UK) by Neubauer hemocytometer (Wertheim, Germany).

\subsection{1-Chlorooctadecane}

1-Chlorooctadecane $\left(\mathrm{CH}_{3}\left(\mathrm{CH}_{2}\right) 16 \mathrm{CH}_{2} \mathrm{Cl}\right)$ was screened from our preliminary study (data not shown) on the chemical fractionation of Cucumis sativus that showed toxicity against date palm dust mites (data not shown). The identified fraction, 1-Chlorooctadecane, selected for the current study was purchased from Sigma-Aldrich in pure form (Cat \# 238368; CAS Number 3386-33-2) to perform compatibility, toxicity, and synergistic studies. The stock solution of the 1-Chlorooctadecane was prepared by dissolving in ethanol.

\subsection{Compatibility of M. anisopliae Spores with 1-Chlorooctadecane}

The potato dextrose agar plates $(90-\mathrm{mm} \times 15 \mathrm{~mm})$ with different concentrations $(0.80,1.60,2.40$, 3.20 , and $4.00 \mathrm{mg} / \mathrm{mL}$ ) of 1-Chlorooctadecane were prepared as described in detail by a previous study [45] Ten microliter spores suspension of M. anisopliae EBCL 02049 at a single concentration $\left(1 \times 10^{6}\right.$ spores $\left./ \mathrm{mL}\right)$ were pipetted in the center of each PDA-plate. The plates after inoculation were sealed with parafilm. All the plates were incubated in complete darkness at $25 \pm 0.5^{\circ} \mathrm{C} ; 70 \pm 5 \% \mathrm{RH}$. The values of three parameters including sporulation (spores $/ \mathrm{mL})$, germination $(\%)$, and vegetative growth $(\mathrm{mm})$, were recorded in order to calculate biological index (BI) [23]. Ten replicates were prepared likewise separately for each parameter using ten PDA-plates.

In the case of percent germination, petri plates were incubated for $12 \mathrm{~h}$ post-inoculation. Percent germination (GR) of M. anisopliae EBCL 02049 spores was calculated by counting a hundred spores under seven different fields of vision. On the other hand, vegetative growth (VG) and sporulation (SP) was determined after 12-days post-inoculation. Seven perpendicular radial growths (mm) of each fungal culture were measured with the help of a transparent ruler for vegetative growth (VG) determination. Furthermore, a $15 \mathrm{~mm}$ diameter sporulating culture of M. anisopliae EBCL 02049 was taken using $15 \mathrm{~mm}$ sterilized cork borer in order to calculate sporulation with the help of Neubauer hemocytometer using $0.05 \%$ Tween 80 solution, under a compound microscope. All the parameters including sporulation, percent germination, and vegetative growth were separately analyzed by One-way Analysis of Variance, and their means by Fisher's LSD test $(\alpha=0.05)$.

The BI was calculated by adding the values of above-mentioned parameters into the standard formula $(\mathrm{BI}=[47 \times \mathrm{VG}+43 \times \mathrm{SP}+10 \times \mathrm{GR}] / 100)$. As per standard criterion, $\mathrm{BI}>66$ ranked as Compatible interaction, while $\mathrm{BI}<42$ ranked as a toxic interaction. The BI between 42 to 66 is ranked as Moderately Toxic interaction [46]. 
4.5. Laboratory Evaluation of M. anisopliae Spores with 1-Chlorooctadecane in Different Proportions against Date Palm Dust Mites

Concentration-mortality response bioassays were performed in order to find the most potent fungal-toxin interaction. The treatments with different proportions used for leaf-dip bioassays are mentioned in Table 5. Each treatment $(50 \mathrm{~mL})$ sole or in different combinations mentioned in Scheme I, II, III, and IV was prepared separately in a sterilized glass beaker. Pesticide-free leaf-discs of date palm $(7.5 \mathrm{~cm}$ length $\times 4 \mathrm{~cm}$ width) were dipped with the help of a forceps. After air drying, fifty deutonymphs (second nymphal stage) date palm dust mites were transferred with the help of a camel hair brush on these exposed leaf-discs surrounded with wet cotton in a petri dish $(150 \mathrm{~mm} \times 20 \mathrm{~mm})$. Control leaf-discs were dipped in their respective solvent. In case of sole application of spores, $0.05 \%$ tween 80 was used as control treatment, while control treatment for 1-Chlorooctadecane was prepared using ethanol with strength used to prepare stock solution of 1-Chlorooctdecane. Control treatment for mixtures was separately prepared using Tween 80 and ethanol with their respective strengths to dissolve them. All the experimental units were maintained at $28.0 \pm 0.5^{\circ} \mathrm{C}$ with a photoperiod of 16:8 h (L:D). Five replicates were prepared likewise. Mortality data were recorded daily for 10 days post-exposure. Dead mites were transferred into the petri dishes lined with dampened sterile filter paper. Mycosis of the inoculating fungal isolate was confirmed by microscopic examination of the corpses. The Abbott formula was applied in order to correct the treatment mortality data from their respective control mortality data [47]. While each treatment mortality data were used to calculate LC $_{50}$ (Lethal Concentration to impart 50\% mortality of date palm dust mites) by Probit analysis [48]. Furthermore, mortality data after angular transformation were analyzed by Repeated Measures ANOVA, and means by Fisher's LSD test [49]. Interaction of different proportions with multiple concentrations as mentioned in Table 5 was designed mainly to calculate joint toxicity as described by Sun et al. [50].

Table 5. Various bioassay schemes evaluated against date palm dust mites.

\begin{tabular}{|c|c|c|c|c|c|c|c|c|c|}
\hline \multirow{4}{*}{$\begin{array}{l}\text { 1-Chlorooctadecane } \\
(\mathrm{mg} / \mathrm{mL})\end{array}$} & \multicolumn{8}{|c|}{ Interaction Schemes } & \multirow{4}{*}{$\begin{array}{c}\text { M. } \\
\text { anisopliae } \\
(\mathrm{mg} / \mathrm{mL})\end{array}$} \\
\hline & \multirow{2}{*}{\multicolumn{2}{|c|}{$\begin{array}{c}\text { Scheme I } \\
1-\text { Chlorooctadecane } \\
(20 \%) \text { : Spores }(80 \%)\end{array}$}} & \multirow{2}{*}{\multicolumn{2}{|c|}{$\begin{array}{c}\text { Scheme II } \\
1-\text { Chlorooctadecane } \\
(40 \%) \text { : Spores }(60 \%)\end{array}$}} & \multirow{2}{*}{\multicolumn{2}{|c|}{$\begin{array}{c}\text { Scheme III } \\
\text { 1-Chlorooctadecane } \\
(60 \%): \text { Spores }(40 \%)\end{array}$}} & \multirow{2}{*}{\multicolumn{2}{|c|}{$\begin{array}{c}\text { Scheme IV } \\
\text { 1-Chlorooctadecane } \\
(80 \%): \text { Spores }(20 \%)\end{array}$}} & \\
\hline & & & & & & & & & \\
\hline & $\begin{array}{l}\text { Toxin } \\
\mathrm{mg} / \mathrm{mL}\end{array}$ & $\begin{array}{l}\text { Spores } \\
\mathrm{mg} / \mathrm{mL}\end{array}$ & $\begin{array}{l}\text { Toxin } \\
\mathrm{mg} / \mathrm{mL}\end{array}$ & $\begin{array}{l}\text { Spores } \\
\mathrm{mg} / \mathrm{mL}\end{array}$ & $\begin{array}{l}\text { Toxin } \\
\mathrm{mg} / \mathrm{mL}\end{array}$ & $\begin{array}{l}\text { Spores } \\
\mathrm{mg} / \mathrm{mL}\end{array}$ & $\begin{array}{l}\text { Toxin } \\
\mathrm{mg} / \mathrm{mL}\end{array}$ & $\begin{array}{l}\text { Spores } \\
\mathrm{mg} / \mathrm{mL}\end{array}$ & \\
\hline 2.4 & 0.48 & 9.6 & 0.96 & 7.2 & 1.44 & 4.8 & 1.92 & 2.4 & 12 \\
\hline 3.2 & 0.64 & 12.8 & 1.28 & 9.6 & 1.92 & 6.4 & 2.56 & 3.2 & 16 \\
\hline 4 & 0.8 & 16 & 1.6 & 12 & 2.4 & 8 & 3.2 & 4 & 20 \\
\hline
\end{tabular}

\subsection{Exploration of Host Antioxidant Defense Response}

Second nymphal stage date palm dust mites infected separately with different treatments mentioned in Table 5 were allowed to feed on date palm leaf-discs at $28.0 \pm 0.5^{\circ} \mathrm{C}$ with a photoperiod of 16:8 h (L:D). Five replicates were prepared. Samples of live date palm dust mites were taken after $24 \mathrm{~h}, 72 \mathrm{~h}$, and $120 \mathrm{~h}$ in order to calculate CAT and SOD activities. Each sample was individually homogenized in ice-cold potassium phosphate buffer saline. The tissues were crushed by a glass homogenizer. All the samples were centrifuged $(12,000 \times g)$ for $15 \mathrm{~min}$. The supernatants were served as enzyme source and taken for protein quantification by a standard method [51]. The enzymatic responses of CAT (Cat \# CAT100-1KT, Sigma-Aldrich, London, UK), and SOD (Cat \# 19160-1KT-F, Sigma-Aldrich, London, UK), upon exposure with different treatments were calculated using the standard protocols provided by the manufacturer of above-mentioned kits. The enzymatic activities in each treatment were expressed in percentages, which are relative to the enzymatic activities data obtained from their respective control treatments. Two-factor factorial analysis was used to analyze 
each treatment relative to control treatment enzymatic activities of date palm dust mites, and their significant differences among different treatments by Fisher's LSD test [52].

\section{Conclusions}

In conclusion, bioassay results of the present study demonstrated that M. anisopliae EBCL 02049 was found to be compatible with all tested concentrations of 1-Chlorooctadecane by showing a very high value of biological index $(\mathrm{BI}>82)$. Our results demonstrated that combined application of $M$. anisopliae spores with 1-Chlorooctadecane resulted in enhanced treatment efficacy against second nymphal stage of date palm dust mites. However, we found that higher proportion of 1-Chlorooctadecane $(80 \%)$ and lower proportion of M. anisopliae spores $(20 \%)$ greatly improved the synergism (joint toxicity $=713$ ). Furthermore, their synergism led to the most potent interaction, which reduced the time and concentration to cause high mortality among date palm dust mites by altering their antioxidant defense mechanism. Hence, the joint action of M. anisopliae spores and 1-Chlorooctadecae could be a promising component of integrated management of date palm dust mites.

Author Contributions: A.H. and A.M.A. conceived the idea. A.H. and A.M.A designed the experiments. A.M.A. provided the resources. A.H. performed the experiments. A.H. and A.M.A. analyzed the data. A.H. wrote the original draft. A.H. and A.M.A. revised and edited the manuscript. A.H. and A.M.A. approved the manuscript for publication. All authors have read and agreed to the published version of the manuscript.

Funding: This research was funded by King Abdul Aziz City for Science and Technology, Kingdom of Saudi Arabia, grant number AT-34-293.

Acknowledgments: We greatly appreciate the assistance provided by some of our colleagues.

Conflicts of Interest: The authors declare no conflict of interest. The funders had no role in the design of the study; in the collection, analyses, or interpretation of data; in the writing of the manuscript, or in the decision to publish the results.

\section{References}

1. Diallo, H. The role of date palm in combat desertification. In The Date Palm: From Traditional Resource to Green Wealth; Emirates Centre for Strategic Studies and Research: Abu Dhabi, UAE, 2005; pp. 13-19.

2. Bashah, M. Date Varieties in the Kingdom of Saudi Arabia. Guidance Booklet: Palms and Dates; King Abdulaziz University Press: Riyadh, Saudi Arabia, 1996.

3. El-Hadrami, I.; El-Hadrami, A. Breeding Date Palm. In Breeding Plantation Tree Crops: Tropical Species; Springer: New York, NY, USA, 2009; pp. 191-216.

4. FAOSTAT Food and Agricultural Commodities Production. Available online: http://www.fao.org/faostat/en/ \#data/QC/visualize (accessed on 26 February 2020).

5. Chaaban, S.B.; Chermiti, B.; Kreiter, S. Comparative demography of the spider mite, Oligonychus afrasiaticus, on four date palm varieties in Southwestern Tunisia. J. Insect Sci. 2011, 11, Article 136. [CrossRef]

6. Amer, S.A.A.; Refaat, A.M.; Momen, F.M. Repellent and oviposition-deterring activity of Rosemary and Sweet Marjoram on the spider mites Tetranychus urticae and Eutetranychus orientalis (Acari: Tetranychidae). Acta Phytopathol. Entomol. Hungarica 2001, 36, 155-164.

7. Reddy, G.; Srinivasa, N.; Muralidhara, M.S. Potentiality of Cinnamomum extracts to two spotted spider mite, Tetranychus urticae Koch and its predator Neoseiulus longispinosus (Evans). J. Biopestic. 2014, 7, 1-11.

8. Chiasson, H.; Bélanger, A.; Bostanian, N.; Vincent, C.; Poliquin, A. Acaricidal properties of Artemisia absinthium and Tanacetum vulgare (Asteraceae) essential oils obtained by three methods of extraction. J. Econ. Entomol. 2001, 94, 167-171. [CrossRef]

9. Idrees, A.; Qasim, M.; Ali, H.; Qadir, Z.A.; Idrees, A.; Qing, J. Acaricidal potential of some botanicals against the stored grain mites, Rhizoglyphus tritici. J. Entomol. Zool. Stud. 2016, 4, 611-617.

10. Elkertati, M.; Blenzar, A.; Jotei, A.B.; Belkoura, I.; Tazi, B. Acaricide effect of some extracts and fractions on Tetranychus urticae Koch (Acari: Tetranychidae). African J. Agric. Res. 2013, 8, 2970-2976.

11. Bussaman, P.; Sa-uth, C.; Rattanasena, P.; Chandrapatya, A. Effect of crude plant extracts on Mushroom mite, Luciaphorus sp. (Acari: Pygmephoridae). Psyche A J. Entomol. 2012, 2012, Article ID 150958. [CrossRef] 
12. Miresmailli, S.; Bradbury, R.; Isman, M.B. Comparative toxicity of Rosmarinus officinalis L. essential oil and blends of its major constituents against Tetranychus urticae Koch (Acari: Tetranychidae) on two different host plants. Pest Manag. Sci. 2006, 62, 366-371. [CrossRef]

13. Hussain, A.; Tian, M.Y.; He, Y.R.; Ahmed, S. Entomopathogenic fungi disturbed the larval growth and feeding performance of Ocinara varians (Lepidoptera: Bombycidae) larvae. Insect Sci. 2009, 16, 511-517. [CrossRef]

14. Hussain, A.; Tian, M.Y.; He, Y.R.; Lei, Y.Y. Differential fluctuation in virulence and VOC profiles among different cultures of entomopathogenic fungi. J. Invertebr. Pathol. 2010, 104, 166-171. [CrossRef]

15. Hussain, A.; Tian, M.Y.; Wen, S.-Y. Proteomic analysis of Formosan Subterranean Termites during exposure to entomopathogenic fungi. Curr. Proteomics 2018, 15, 229-240. [CrossRef]

16. Hussain, A.; Rizwan-ul-haq, M.; AlJabr, A.M.; Al-Ayedh, H. Host-pathogen interaction for screening potential of Metarhizium anisopliae isolates against the date-palm dust mite, Oligonychus afrasiaticus (McGregor) (Acari: Tetranychidae). Egypt. J. Biol. Pest Control 2019, 29, 63. [CrossRef]

17. Kristiani, E.B.E.; Nugroho, L.H.; Moeljopawiro, S.; Widyarini, S. Characterization of volatile compounds of Albertisia papuana Becc root extracts and cytotoxic activity in breast cancer cell line T47D. Trop. J. Pharm. Res. 2016, 15, 959-964. [CrossRef]

18. Swami, S.B.; Thakor, N.S.J.; Patil, M.M.; Haldankar, P.M. Jamun (Syzygium cumini (L.)): A review of its food and medicinal uses. Food Nutr. Sci. 2012, 3, 1100-1117.

19. Li, G.; Jiang, Y.; Li, Y.; He, T.; Wang, Y.; Ji, T.; Zhai, W.; Zhao, L.; Zhou, X. Analysis and biological evaluation of Arisaema amuremse Maxim essential oil. Open Chem. 2019, 17, 647-654. [CrossRef]

20. Siddiquee, S.; Cheong, B.E.; Taslima, K.; Kausar, H.; Hasan, M.M. Separation and identification of volatile compounds from liquid cultures of Trichoderma harzianum by GC-MS using three different Capillary Columns. J. Chromatogr. Sci. 2012, 50, 358-367. [CrossRef]

21. Fetoh, B.; Al-Shammery, K. Acaricidal ovicial and repellent activities of some plant extracts on the date palm dust mite, Oligonychus afrasiaticus Meg. (Acari: Tetranychidae). Int. J. Environ. Sci. Eng. 2011, 2, 45-52.

22. Lakhdari, W.; Dehliz, A.; Acheuk, F.; Soud, A.; Hammi, H.; Mlik, R.; Doumandji-Mitiche, B. Acaricidal activity of aqueous extracts against the mite of date palm Oligonychus afrasiaticus Meg (Acari: Tetranychidae). J. Med. Plants 2015, 3, 113-117.

23. AlJabr, A.; Hussain, A.; Rizwan-ul-haq, M. Toxin-Pathogen synergy reshaping detoxification and antioxidant defense mechanism of Oligonychus afrasiaticus (McGregor). Molecules 2018, 23, 1978. [CrossRef]

24. Hussain, A.; Rizwan-ul-Haq, M.; AlJabr, A.M.; Al-Ayedh, H. Evaluation of host-pathogen interactions for selection of entomopathogenic fungal isolates against Oligonychus afrasiaticus (McGregor). BioControl 2020, 65, 185-195. [CrossRef]

25. Shi, W.-B.; Zhang, L.; Feng, M.-G. Time-concentration-mortality responses of carmine spider mite (Acari: Tetranychidae) females to three hypocrealean fungi as biocontrol agents. Biol. Control 2008, 46, 495-501. [CrossRef]

26. Gonçalves Diniz, A.; Barbosa, L.F.S.; da Silva Santos, A.C.; de Oliveira, N.T.; da Costa, A.F.; Carneiro-Leão, M.P.; Tiago, P.V. Bio-insecticide effect of isolates of Fusarium caatingaense (Sordariomycetes: Hypocreales) combined to botanical extracts against Dactylopius opuntiae (Hemiptera: Dactylopiidae). Biocontrol Sci. Technol. 2020, 30, $1-12$.

27. Wraight, S.P.; Filotas, M.J.; Sanderson, J.P. Comparative efficacy of emulsifiable-oil, wettable-powder, and unformulated-powder preparations of Beauveria bassiana against the melon aphid Aphis gossypii. Biocontrol Sci. Technol. 2016, 26, 894-914. [CrossRef]

28. Hernández, M.M.; Martínez-Villar, E.; Peace, C.; Pérez-Moreno, I.; Marco, V. Compatibility of the entomopathogenic fungus Beauveria bassiana with flufenoxuron and azadirachtin against Tetranychus urticae. Exp. Appl. Acarol. 2012, 58, 395-405. [CrossRef]

29. Hussain, A.; Rizwan-ul-Haq, M.; Al-Ayedh, H.; AlJabr, A. Susceptibility and immune defence mechanisms of Rhynchophorus ferrugineus (Olivier) (Coleoptera: Curculionidae) against entomopathogenic fungal infections. Int. J. Mol. Sci. 2016, 17, 1518. [CrossRef]

30. Hussain, A.; Ahmed, S.; Shahid, M. Laboratory and field evaluation of Metarhizium anisopliae var. anisopliae for controlling subterranean termites. Neotrop. Entomol. 2011, 40, 244-250.

31. Hussain, A. Reprogramming the virulence: Insect defense molecules navigating the epigenetic landscape of Metarhizium robertsii. Virulence 2018, 9, 447-449. [CrossRef] 
32. Hussain, A.; Rizwan-ul-Haq, M.; Al-Ayedh, H.; Al-Jabr, A. Mycoinsecticides: Potential and future perspective. Recent Pat. Food. Nutr. Agric. 2014, 6, 45-53. [CrossRef]

33. Hussain, A.; Tian, M.Y. Germination pattern and inoculum transfer of entomopathogenic fungi and their role in disease resistance among Coptotermes formosanus (Isoptera: Rhinotermitidae). Int. J. Agric. Biol. 2013, 15, 319-324.

34. Wink, M. Plant secondary metabolites modulate insect behavior-steps toward addiction? Front. Physiol. 2018, 9, 364. [CrossRef]

35. Wink, M. Interference of alkaloids with neuroreceptors and ion channels. Stud. Nat. Prod. Chem. 2000, $21,3-122$.

36. AlJabr, A.M.; Hussain, A.; Rizwan-ul-Haq, M.; Al-Ayedh, H. Toxicity of Plant Secondary Metabolites Modulating Detoxification Genes Expression for Natural Red Palm Weevil Pesticide Development. Molecules 2017, 22, 169. [CrossRef] [PubMed]

37. Hussain, A.; Rizwan-ul-haq, M.; AlJabr, A.M.; Al-Ayedh, H. Lethality of Sesquiterpenes Reprogramming Red Palm Weevil Detoxification Mechanism for Natural Novel Biopesticide Development. Molecules 2019, 24, 1648. [CrossRef] [PubMed]

38. Hussain, A.; Rizwan-ul-Haq, M.; Al-Ayedh, H.; Aljabr, A.M. Toxicity and detoxification mechanism of black pepper and its major constituent in controlling Rhynchophorus ferrugineus Olivier (Curculionidae: Coleoptera). Neotrop. Entomol. 2017, 46, 685-693. [CrossRef]

39. Sáenz-de-Cabezón Irigaray, F.J.; Marco-Mancebón, V.; Pérez-Moreno, I. The entomopathogenic fungus Beauveria bassiana and its compatibility with triflumuron: Effects on the twospotted spider mite Tetranychus urticae. Biol. Control 2003, 26, 168-173. [CrossRef]

40. Zou, C.; Li, L.; Dong, T.; Zhang, B.; Hu, Q. Joint action of the entomopathogenic fungus Isaria fumosorosea and four chemical insecticides against the whitefly Bemisia tabaci. Biocontrol Sci. Technol. 2014, 24, 315-324. [CrossRef]

41. Akbar, W.; Lord, J.C.; Nechols, J.R.; Loughin, T.M. Efficacy of Beauveria bassiana for red flour beetle when applied with plant essential oils or in mineral oil and organosilicone carriers. J. Econ. Entomol. 2005, 98, 683-688. [CrossRef]

42. Lu, F.; Liang, X.; Lu, H.; Li, Q.; Chen, Q.; Zhang, P.; Li, K.; Liu, G.; Yan, W.; Song, J.; et al. Overproduction of superoxide dismutase and catalase confers cassava resistance to Tetranychus cinnabarinus. Sci. Rep. 2017, 7, 40179. [CrossRef]

43. Deisseroth, A.; Dounce, A.L. Catalase: Physical and chemical properties, mechanism of catalysis, and physiological role. Physiol. Rev. 1970, 50, 319-375. [CrossRef]

44. Hussain, A.; Tian, M.Y.; He, Y.R.; Ruan, L.; Ahmed, S. In vitro and in vivo culturing impacts on the virulence characteristics of serially passed entomopathogenic fungi. J. Food Agric. Environ. 2010, 8, 481-487.

45. Hussain, A.; Tian, M.-Y.; Wen, S.-Y. Exploring the caste-specific multi-layer defense mechanism of Formosan Subterranean Termites, Coptotermes formosanus Shiraki. Int. J. Mol. Sci. 2017, 18, 2694. [CrossRef] [PubMed]

46. Schumacher, V.; Poehling, H.-M. In vitro effect of pesticides on the germination, vegetative growth, and conidial production of two strains of Metarhizium anisopliae. Fungal Biol. 2012, 116, 121-132. [CrossRef]

47. Abbott, W.S. A method of computing the effectiveness of an insecticide. J. Econ. Entomol. 1925, 18, $265-267$. [CrossRef]

48. StatsDirect StatsDirect Statistical Software. England. StatsDirect Ltd., 2013. Available online: http: //www.statsdirect.com (accessed on 25 February 2020).

49. Statistix Statistix 8.1; Analytical Software: Tallahassee, FL, USA, 2003.

50. Sun, Y.-P.; Johnson, E.R. Analysis of joint action of insecticides against House flies. J. Econ. Entomol. 1960, 53, 887-892. [CrossRef]

51. Bradford, M.M. A rapid and sensitive method for the quantitation of microgram quantities of protein utilizing the principle of protein-dye binding. Anal. Biochem. 1976, 72, 248-254. [CrossRef]

52. SAS Institute SAS User's Guide: Statistics; SAS Institute: Cary, NC, USA, 2000.

Sample Availability: Samples of the compound, 1-Chlorooctadecane and Metarhizium anisopliae EBCL 02049 are available from the authors. 